\title{
La représentation des extraterrestres ${ }^{1}$
}

\section{The depiction of aliens}

\author{
Roland Lehoucq ${ }^{1}$ \\ ${ }^{1}$ Astrophysicien au CEA
}

RÉSUMÉ. L'humanité a toujours cherché à se définir et s'est bien sûr penchée sur la question de l'altérité. Dès que la notion de pluralité des mondes a été posée, notamment par Giordano Bruno à la fin du XVI ${ }^{\mathrm{e}}$ siècle, ou par le marquis de Fontenelle à la fin du XVII siècle, la question de la vie, voire de l'intelligence, extra-terrestre l'a naturellement prolongée. La figure de l'extraterrestre interroge la conception que nous avons de l'unité du vivant, ou de son infinie diversité, et que les fictions qui le mettent en scène reflètent plutôt le regard que nous portons sur nous-mêmes.

ABSTRACT. Humanity has always sought to define itself and has of course considered the question of alterity. As soon as the notion of the plurality of worlds was posed, notably by Giordano Bruno at the end of the sixteenth century, or by the Marquis de Fontenelle at the end of the seventeenth century, the question of extraterrestrial life, and even intelligence, naturally followed. The figure of the extraterrestrial questions our conception of the unity of living species, or of their infinite diversity, and that the fictions that feature them rather reflect the way we look at ourselves.

MOTS-CLÉS. extraterrestre, science-fiction, formes du vivant, biodiversité.

KEYWORDS. extraterrestrials, science fiction, living forms, biodiversity.

La toute première représentation d'un être extraterrestre semble remonter aux années 1835-1836². Il s'agit d'hommes chauve-souris habitant la Lune, observés au moyen d'un super télescope imaginaire. Mais l'extraterrestre le plus souvent représenté est sans doute le Martien, créature imaginaire originaire de la planète Mars. L'un des plus célèbre est celui de Frank Paul, illustrant la couverture de la revue Science and Invention datée d'août 1924. Cette représentation se veut conforme aux connaissances que les scientifiques avaient des conditions martiennes : l'être a de grandes oreilles pour capter les ondes sonores dans une atmosphère raréfiée, un nez et des yeux rétractables à cause des basses températures, un torse volumineux adapté à une atmosphère pauvre en oxygène et des pieds-ventouses pour bien adhérer au sol d'une planète à faible gravité. Ce Martien est aussi doté d'une double antenne télépathique et d'un désintégrateur atomique... Par la suite, les Martiens seront souvent représentés comme des êtres humanoïdes, minces, avec une grosse tête et des yeux globuleux. Ils sont aussi souvent de couleur verte, d'où leur surnom de «petits hommes verts ». Cette couleur pourrait avoir son origine dans un roman d'Edgar Rice Burroughs, le père de Tarzan, intitulé A Princess of Mars (1912). L'auteur y décrit différentes espèces de Martiens, dont l'une a la peau verte. Cette couleur sera reprise par plusieurs autres auteurs et fera parfois le titre de leur ouvrage, comme Harold Sherman dans The Green Man (1946) ou encore Damon Knight dans The Third Little Green Man (1947). Dans la tradition des contes, la couleur verte est omniprésente pour évoquer certaines créatures féeriques ou fantomatiques.

Avec leur arrivée au cinéma, l'aspect des extraterrestres s'est, en apparence, considérablement diversifié. Face à cette profusion d'espèces, Denis Van Waerebeke a réalisé un court-métrage humoristique intitulé Classification systématique du vivant extraterrestre, dans lequel il applique aux extraterrestres du cinéma les règles de classification appliquées au vivant terrestre ${ }^{3}$. Il en ressort

\footnotetext{
${ }^{1}$ Cet article est une version remaniée d'une publication initialement publiée en novembre 2011 dans la revue Médecine Science

${ }^{2}$ Y. Bosson et F. Abdelouahab, Dictionnaire visuel des mondes extraterrestres, Flammarion, 2010.

${ }^{3}$ Ce court métrage est visible en streaming sur le site http://www.6nema.com/cargo/court-

metrage/classification_systematique_du_vivant_extraterrestre-1043
} 
notamment que la forme humanoïde domine largement. C'est assez logique si l'on considère que l'acteur qui joue le rôle de la créature doit pouvoir enfiler son costume sans trop limiter ses mouvements. Cette contrainte aurait dû disparaître avec l'utilisation d'effets spéciaux ou d'acteurs virtuels (motion capture). Mais les réalisateurs ne s'inspirent guère de la biodiversité terrestre qui réserve pourtant des surprises étonnantes. Malheureusement, nous ne nous intéressons pas aux êtres vivants chez lesquels une symétrie bilatérale, une tête ou des yeux ne nous apparaissent pas spontanément. C'est donc la presque totalité du vivant terrestre qui est purement et simplement ignorée quand il s'agit d'imaginer des extraterrestres vraiment originaux ${ }^{4}$. De ce point de vue, le film Avatar (2009) est emblématique. Son réalisateur, James Cameron, dote le monde où se déroule l'histoire - Pandora - d'un écosystème très semblable au nôtre : on y trouve des vertébrés, des carnivores, des herbivores, des arbres, des fleurs, et bien sûr des extraterrestres anthropomorphes, les $\mathrm{Na}$ 'vis.

S'il y a tant de créatures familières sur Pandora c'est pour donner aux spectateurs un exotisme non déroutant. Le détournement de l'extraordinaire diversité du vivant terrestre facilite la tâche : l'hélicoradians, une plante forestière de Pandora, ressemble aux spirales de tentacules colorées du vers marin Spirobrancheus giganteus, le direhorse est une copie du cheval bien qu'il soit hexapode et se nourrisse avec une trompe comme un papillon, le titanothérium est manifestement une chimère de rhinocéros et de requin marteau, le banshee a un air de ptérosaure mais son système respiratoire est semblable à celui du dauphin. Dans un monde ni trop différent du nôtre, ni trop familier, nous succombons à la fascination de l'étrange tout en préservant l'empathie. Mais rien ne garantit qu'il y ait des similitudes entre une éventuelle vie extraterrestre et la nôtre : aucune certitude qu'elle prenne la forme d'une vie cellulaire (unité fondamentale du vivant terrestre), qu'elle soit à base d'ADN (hypothèse indispensable pour créer l'avatar de Jake Scully en combinant le sien à celui d'un Na'vi) ou qu'un être intelligent soit humanoïde. Les images d'Avatar posent donc la question de l'unité des formes et de l'émergence d'une vie extraterrestres Sur une planète semblable à la Terre, les êtres vivants seront-ils similaires à ceux que nous connaissons ? L'évolution des espèces y suivra-t-elle les voies terrestres ou bien d'autres radicalement différentes ? L'apparition de la vie sur Terre étaitelle inévitable ou bien n'était-ce qu'un coup de chance ? Que se passerait-il si l'on pouvait rejouer toute l'évolution terrestre depuis l'apparition des premières formes de vie ? Faute d'avoir déjà découvert une activité biologique actuelle ou fossile hors de la Terre, ces questions restent pour l'instant sans réponses : certains biologistes, comme Stephen J. Gould, estiment que si l'évolution terrestre était rejouée pratiquement rien ne serait semblable ${ }^{5}$ tandis que d'autres, comme Simon C. Morris, affirment que des structures morphologiques et métaboliques se répèteraient ${ }^{6}$.

Certaines propriétés de la vie sont dues au hasard: à chaque génération, des mutations se produisent de façon aléatoire au sein de l'ADN ; parmi ces mutations, certaines permettent de mieux survivre dans les conditions où elles se produisent. Par le jeu de la reproduction ces mutations avantageuses finissent par envahir les populations d'êtres vivants. Mais si l'évolution comporte une part de hasard (les mutations), elle a aussi une part de nécessité : les mutations avantageuses le sont parfois pour des raisons physiques profondes. Les exemples ne manquent pas. Ainsi, la forme d'un arbre (un fort axe vertical, le tronc, qui plonge des racines dans le sol et supporte des appendices secondaires, les branches) est une réponse universelle à une contrainte environnementale : haute taille pour gagner la course à la lumière tout en restant bien ancré dans le sol pour y puiser les nutriments. Cette structure se trouvait déjà sur les fougères arborescentes ou les calamites du Carbonifère, il y a 300 millions d'années (Ma). Dans le règne animal on dénombre une quinzaine de

\footnotetext{
${ }^{4}$ Voir l'article de G. Lecointre " Y a-t-il des extraterrestres dans mon assiette ? ", in Sciences et science fiction, livre dirigé par U. Bellagamba, P. Gyger, R. Lehoucq et C. Pieyre, éditions La Martinière, 2010.
}

${ }^{5}$ S. J. Gould, Wonderful life: the burgess shale and the nature of history, W. W. Norton, 1989.

${ }^{6}$ S. C. Morris, Life's solution: inevitable humans in a lonely universe. Cambridge University Press, 2003. 
type d'yeux utilisant divers procédés de détection de la lumière et de formation d'une image. Les organes de vol se retrouvent chez les insectes, les poissons (exocet), les ptérosaures (qui vécurent du Trias, -230 Ma, au Crétacé, -65 Ma), les oiseaux (descendants des dinosaures) et les mammifères (comme la chauve-souris). La forme hydrodynamique et les nageoires sont aussi apparues plusieurs fois de façon totalement indépendante : bien évidemment chez les poissons mais aussi chez les ichtyosaures (des reptiles marins qui vécurent de -250 Ma à -90 Ma), les oiseaux aquatiques comme le manchot, les mammifères aquatiques (comme les otaries), les cétacés (comme le grand dauphin) et les siréniens (comme le dugong). Cette évolution morphologique analogue est liée au fait que la force exercée par l'eau sur un corps en mouvement est la plus faible possible pour des profils en forme d'ogive. Soumises à cette même contrainte, ces espèces ont, chacune de leur côté, évolué vers une solution adaptative très similaire au problème du déplacement dans un milieu dense comme l'eau. Ainsi, des solutions similaires favorisant la survie sont apparus dans diverses branches du buisson évolutif terrestre car des contraintes physiques peuvent imposer une relation entre la fonction d'un organe et sa forme, entre la niche écologique d'une espèce et les organes qu'elle porte. Cette évolution convergente est le produit de l'évolution d'espèces soumises aux mêmes lois physiques et aux mêmes contraintes environnementales ${ }^{7,8}$.

S'il existe une vie extraterrestre, elle résultera sans doute, comme sur Terre, de milliards d'années d'évolution et pourraient bien avoir quelques traits communs avec la vie terrestre car les lois physiques régissant la matière et les processus qui la transforment sont universelles. Ainsi, la vie terrestre est essentiellement composée de carbone, d'hydrogène, d'oxygène et d'azote (avec une pincée de phosphore et de soufre) qui sont aussi quatre éléments chimiques très abondants dans l'univers'. Il est donc raisonnable de penser qu'une vie extraterrestre sera aussi basée sur ces éléments. D'autres part, du microorganisme le plus simple à l'organisme le plus complexe, on retrouve un certain nombre de fonctions vitales de base :

- se mouvoir dans un liquide, dans un gaz, sur une surface, en volume, bref dans un milieu plus ou moins dense, en étant éventuellement soumis à la gravité ;

- échanger avec son milieu, pour se nourrir, rejeter des déchets ou respirer ;

- interagir avec son milieu pour s'en informer - à propos de nourriture, de prédateur ou de proie avec des sens tactile, olfactif, auditif, visuel, électrique, magnétique ou autre ;

- échanger de l'information avec ses congénères grâce à des organes de communication, tant en émission qu'en réception, eux aussi fondés sur les sens tactile, olfactif, auditif, visuel, électrique ou magnétique.

Dans ce cadre, les possibilités sont nombreuses. Il ne paraît donc guère facile de démontrer que des êtres extraterrestres ressembleront aux êtres terrestres, mais l'hypothèse d'une diversité radicale n'est pas non plus assurée. Alors que certains exobiologistes insistent sur le fait que la vaste biodiversité terrestre suggère qu'elle le sera encore plus dans l'espace, d'autres font remarquer que la convergence évolutive pourrait bien imposer de nombreuses similarités entre les vies terrestres et extraterrestres. La difficulté réside dans le fait que, n'ayant qu'un seul exemple sous la main, il est facile de tomber dans une sorte de «chauvinisme terrestre » en imaginant les formes que pourrait prendre une vie extraterrestre. De ce point de vue, «vie» ne signifie évidemment pas seulement « vie intelligente » ou « vie multicellulaire vertébrée ». Ces formes-là ne représentent qu'une infime

\footnotetext{
${ }^{7}$ C. Pickover, The science of aliens, Basic Books, 2003.
}

${ }^{8}$ J. Cohen et I. Stewart, What Does a Martian Look Like: The Science of Extraterrestrial Life, Ebury Press, 2004.

${ }^{9}$ E. Anders \& M. Ebihara, Solar-system abundances of the elements, Geochimica et Cosmochimica Acta 46, 2363 (1982). 
fraction de la biodiversité terrestre ${ }^{10}$ et ne sont peut-être pas universelles. La vie terrestre a commencé par des êtres unicellulaires et la plupart des paléontologues estiment qu'elle existe depuis au moins 3,4 milliards d'années ${ }^{11}$. Le plus vieil organisme multicellulaire n'a fait son apparition qu'il y a 1,2 milliards d'années ${ }^{12}$. Si l'on découvre un jour une vie extraterrestre, il est possible qu'elle soit microscopique. Et une vie multicellulaire n'a pas forcément besoin d'un squelette même si cette structure est commune à la grande majorité des animaux terrestres pour lesquels la gravité joue un rôle. Sur Terre, les plus anciens fossiles de vertébrés datent de 530 millions d'années (Cambrien) $)^{13}$ et ce groupe ne représente aujourd'hui que $5 \%$ des espèces terrestres. Si l'on rejouait l'histoire de la vie sur Terre, il est possible que les vertébrés n'apparaissent jamais.

Pour conclure, notons que les représentations d'extraterrestres sont toujours le reflet de leur époque. Ainsi, en 1838, le naturaliste Pierre Boitard publie une série d'articles sur les habitants de chaque planète du système solaire, imprégnée par les théories évolutionnistes et le colonialisme ambiant. Plus tard, c'est lors d'une discussion au sujet des conséquences dramatiques de l'arrivée des Anglais en Tasmanie ${ }^{14}$, qu'une réflexion de son frère Frank aurait donné l'idée à Herbert Wells d'écrire La Guerre des Mondes (1897), qui fit des Martiens nos ennemis extraterrestres les plus dangereux. Les êtres que décrit Wells, grands cerveaux dotés de huit paires de tentacules et incapables de se déplacer par eux-mêmes, sont peut-être une métaphore de l'humain du futur, au stade ultime de son évolution: un être-cerveau, dépourvu d'organes locomoteurs, digestifs et reproducteurs, uniquement dotés de «mains » à titre de prolongement naturel du cerveau. Cette altérité de l'extraterrestre permet à certains auteurs de mener la quête philosophique de la spécificité de notre humanité. Qu'est-ce qui définit l'humain dans l'infini des possibles ? En nous faisant voyager sur des mondes qui n'existent pas à la rencontre d'êtres imaginaires, la science-fiction nous parle surtout de nous, de nos vertiges face à notre insignifiante position dans l'Univers et des constructions que nous échafaudons, au fil du temps, pour nous en accommoder.

\footnotetext{
${ }^{10}$ Y. M. Bar-On, R. Phillips and R. Milo, The biomass distribution on Earth, PNAS 115 (25), 6506-6511 (2018).

${ }^{11}$ D. Wacey et al., Microfossils of sulphur-metabolizing cells in 3.4-billion-year-old rocks of Western Australia, Nature Geoscience (2011), http://www.nature.com/ngeo/journal/vaop/ncurrent/full/ngeo1238.html

${ }^{12}$ N. J. Butterfield, Bangiomorpha pubescens: implications for the evolution of sex, multicellularity, and the Mesoproterozoic/Neoproterozoic radiation of eukaryotes, Paleobiology 26, 386-404 (2000).

${ }^{13}$ D. G. Shu et al., Lower Cambrian vertebrates from south China, Nature 402, 42-46 (1999).

${ }^{14} \mathrm{Au} \mathrm{XVIII}$ ' siècle, les indigènes de l'île de Tasmanie, au large de l'Australie, furent victimes des colons anglais quand les soldats de Sa Majesté firent de l'île une colonie pénitentiaire. En 1876, tous avaient été exterminés.
} 